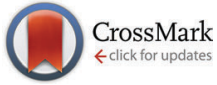

Cite this: Phys. Chem. Chem. Phys., 2015, 17, 7907

Received 29th December 2014 Accepted 7th February 2015

DOI: $10.1039 / \mathrm{c} 4 \mathrm{cp} 06085 \mathrm{~g}$

www.rsc.org/pccp

\title{
Tailored interface and enhanced elastic modulus in epoxy-based composites in presence of branched poly(ethyleneimine) grafted multiwall carbon nanotubes
}

\author{
Rani Rohini and Suryasarathi Bose* \\ In this study, branched poly(ethyleneimine), BPEl, was synthesized from carboxylic acid terminated \\ multi-walled carbon nanotubes (c-MWNTs) and characterized using FTIR, TEM and TGA. The BPEI was \\ then chemically grafted onto MWNTs to enhance the interfacial adhesion with the epoxy matrix. The \\ epoxy composites with c-MWNTs and the BPEI-g-MWNTs were prepared using a sonication and \\ mechanical stirring method, followed by curing at $100{ }^{\circ} \mathrm{C}$ and post-curing at $120{ }^{\circ} \mathrm{C}$. The dynamic \\ mechanical thermal analysis showed an impressive $49 \%$ increment in the storage elastic modulus in the \\ composites. In addition, the nanoindentation on the composites exhibited significant improvement in \\ the hardness and decrease in the plasticity index in the presence of the BPEI-g-MWNTs. Thus, epoxy \\ composites with BPEI-g-MWNTs can be further explored as self-healing materials.
}

\section{Introduction}

Ever since the discovery of carbon nanotubes (CNTs) in 1991 by Iijima, they have attracted a great deal of interest among researchers from both academia and industry. This is owing to their unique combination of mechanical, electrical, thermal and EMI shielding properties as well as due to their high aspect ratio. ${ }^{1-8}$ Ajayan first reported the development of CNT-polymer nanocomposites to enhance and manipulate different properties of polymeric materials. ${ }^{9}$ The fibrous-like structure, high aspect ratio and low density of CNTs make them suitable candidates as reinforcement agents for polymer based composites. Epoxy, a thermosetting polymer, is a potential material having a 3-D network-like structure. Therefore, it is used in aviation, electronics, thermal insulation, coatings, adhesives and composites industries. ${ }^{10}$ Some challenges such as brittleness, poor crack propagation, and high cross linking density limits its application. In the recent past, different nanoscale reinforcements, such as nanoclay, carbon nanotubes, silica, silver and graphene, have been added to the epoxy matrix to overcome its limitations. ${ }^{10-13}$

A significant volume of work on CNT-Epoxy composites has been carried out in the past decade to understand the load transfer mechanism, thermal response, effect of network formation and interfacial behaviour. Because of the smooth surface of CNTs, efficient load transfer is generally hindered in addition to the challenge associated with agglomerated bundles of nanotubes. ${ }^{14}$

Department of Materials Engineering, Indian Institute of Science, Bangalore560012, India.E-mail: sbose@materials.iisc.ernet.in
Covalent functionalization of CNTs is an effective tool to improve interfacial adhesion between CNTs and epoxy. ${ }^{13-15}$ For instance, when amino functionalized nanotubes were subjected to dicarboxylic acid acyl peroxide treatment, they revealed an impressive $70 \%$ increase in the ultimate strength and storage modulus. ${ }^{14}$ Non-ionic surfactant (Triton) treated CNTs showed bridging effects between nanotubes and epoxy because of the hydrophilic and hydrophobic parts of the surfactant. ${ }^{15}$ TETA grafted CNTs improved the interfacial interaction, and thus the mechanical and thermal properties of epoxy-based composites were improved. ${ }^{13}$ Yoonessi et al. ${ }^{16}$ covalently modified CNTs with aromatic, aliphatic, and aliphatic ether diamines. It was observed that aromatic diamine functionalized CNTs significantly enhanced the glass transition temperature and shear moduli. In another strategy, the epoxy chains were attached to CNTs using poly(acryloyl chloride) as a bridging agent. These composites showed higher glass transition temperature and improved mechanical properties. ${ }^{17}$

Recently, nanoindentation has proved to be an important tool to measure the localized mechanical properties, i.e., modulus, stiffness, hardness, and elastic-plastic behaviour. ${ }^{18}$ It also serves as an efficient tool to study the interface between the thermoset and thermoplastic systems and the diffusion of one phase into another. ${ }^{19}$ In a recent review, the mechanical behaviour of thermoset polymers with different functionalized CNTs has been presented and the factors involved, such as, dispersion and the concentration of filler, have been highlighted. ${ }^{20}$

In the present work, an epoxy matrix has been reinforced with carboxylic acid functionalized MWNTs (c-MWNTs) and 
branched poly(ethyleneimine) grafted MWNTs (BPEI- $g$-MWNTs) to achieve better dispersion and enhanced interfacial adhesion with the epoxy matrix. BPEI is a macromolecule rich in amine and imine groups, which can interact with the epoxy resin. ${ }^{21}$ In one of the earlier studies, carbon nanofibers were amine functionalized using PEI and made into nanopapers to reinforce an epoxy matrix. ${ }^{22}$ Schaubroeck et al. $^{23}$ studied the adhesion properties of PEI anchored epoxy resin coupled via cyanuric chloride on the surface of metals. In a recent work, PEI functionalized CNTs based epoxy composites were fabricated to study their thermal conductivity. ${ }^{24}$ Kim et al. ${ }^{25}$ described the synthesis of a BPEI-GO conjugate for gene delivery applications. Although some studies that highlight the improved properties of epoxy matrix in the presence of BPEI exist, a detailed analysis has not received much attention. Therefore, this work focuses on understanding the mechanical behaviour of epoxy composites in the presence of c-MWNTs and BPEI- $g$-MWNTs by dynamic mechanical thermal analysis and nanoindentation. BPEI was covalently grafted on the surface of c-MWNTs using EDC chemistry. $^{26}$ The resulting product was further characterized using FTIR, TEM, TGA and AFM. Epoxy composites were prepared with c-MWNT and BPEI- $g$-MWNT by sonication and mechanical mixing followed by curing and post-curing cycles. The state of dispersion of MWNTs was studied using SEM. The mechanical properties, i.e., storage modulus, hardness and elastic-plastic behaviour, were studied using DMA and nanoindentation.

\section{Experimental}

\section{Materials and method}

Epoxy resin (bisphenol- $F$-epichlorohydrin, EPOLAM 8052) and amine based hardener (2,2'-dimethyl-4,4'methylene bis(cyclohexylamine)) were kindly provided by Axson technologies (France). The branched poly(ethyleneimine) $\left(M_{\mathrm{n}}=25000\right)$ was procured from Sigma Aldrich. The carboxylic acid functionalized multi-walled carbon nanotubes (c-MWNTs, NC3151) were obtained from Nanocyl, Belgium. Ethanol, 1-ethyl-3-[3-(dimethylamino) propyl] carbodiimide hydrochloride (EDC), $N$-hydroxysuccinimide (NHS) and triethylamine (TEA) were procured commercially.

\section{Characterization}

The covalent functionalization of BPEI onto MWNTs was confirmed using Fourier transform infrared spectroscopy (FTIR) on a PerkinElmer instrument. The morphology of the synthesized BPEI- $g$ MWNT was characterized by transmission electron microscopy (TEM) on FEI Tecnai F30 S-TWIN and the \% grafting was evaluated using Netzsch STA 409 PC Thermogravimetry Analyzer (TGA). The state of dispersion and the fracture morphology of the epoxy composites were observed by scanning electron microscopy (SEM) on FEI Sirion XL30 FEG. The mechanical behaviour of the composites was studied on Hysitron TI 900 TriboIndenter Nanoindentator with Berkovich indenter and 3-point bending clamp of TA Q800 Dynamic Mechanical Analyzer (DMA). Thermal decomposition analysis was done using Netzsch STA 409 PC Thermogravimetry Analyzer (TGA) at the rate of $10{ }^{\circ} \mathrm{C} \mathrm{min}^{-1}$ under oxygen atmosphere.

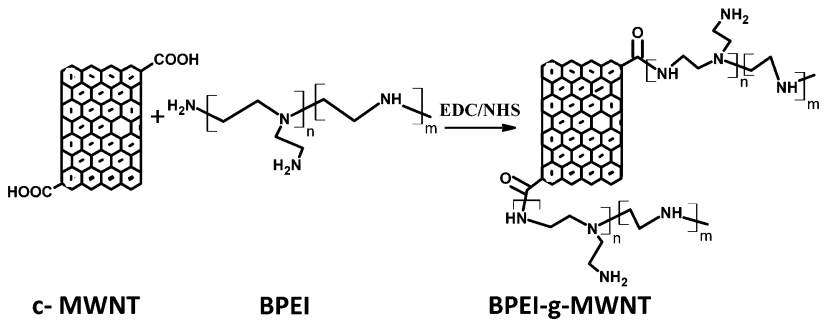

Scheme 1 Synthesis of BPEI-g-MWNTs.

\section{Synthesis of BPEI- $g$-MWNTs}

To immobilize BPEI on the carboxylic acid groups present on the surface of c-MWNTs, the protocol shown in Scheme 1 was adopted. Typically, c-MWNTs $(50 \mathrm{mg})$ were sonicated for 30 mins to disperse in DI $\mathrm{H}_{2} \mathrm{O}(10 \mathrm{ml})$. BPEI $(25 \mathrm{mg})$ was separately dissolved in DI $\mathrm{H}_{2} \mathrm{O}(10 \mathrm{ml})$ and TEA $(200 \mu \mathrm{l})$ was added to this solution. EDC $(250 \mathrm{mg}, 0.16 \mathrm{mmol})$ and NHS (1.49 $\mathrm{mg}, 0.13 \mathrm{mmol}$ ) were also added to this solution and it was transferred to a round-bottomed flask and magnetically stirred at $37{ }^{\circ} \mathrm{C}$. Subsequently, BPEI-TEA solution was added and the resulting mixture was again stirred for $24 \mathrm{~h}$ at $37^{\circ} \mathrm{C}$. Finally, the product was subjected to dialysis to remove unreacted BPEI, vacuum dried and characterized.

\section{Preparation of epoxy composites}

The various epoxy composites were mixed using a mechanical overhead stirrer from Heidolph RZR 2102. The epoxy composites were prepared using 1 wt $\%$ of c-MWNT and BPEI- $g$ MWNT. It is important to note that the concentration of CNTs was fixed by estimating the concentration of BPEI apriori using TGA. A known amount of c-MWNTs and BPEI- $g$-MWNTs were sonicated for $30 \mathrm{~min}$ in ethanol. The epoxy resin was then added to these solutions followed by sonication for $30 \mathrm{~min}$. The resultant solutions were then kept in a vacuum oven at $60{ }^{\circ} \mathrm{C}$ to evaporate the solvent. After solvent removal, these mixtures were mechanically stirred at $400 \mathrm{rpm}$ for $2 \mathrm{~h}$ at $60{ }^{\circ} \mathrm{C}$. The hardener was then added followed by mechanical stirring for $10 \mathrm{~min}$ at $500 \mathrm{rpm}$. The resulting epoxy composites were then poured in a preheated teflon-coated mould and cured at $100{ }^{\circ} \mathrm{C}$ for $1 \mathrm{~h}$ followed by post-curing at $120{ }^{\circ} \mathrm{C}$ for $1 \mathrm{~h}$. Flat disk samples (diameter $10 \mathrm{~mm}$ and thickness $2 \mathrm{~mm}$ ) were used for nanoindentation, while rectangular samples $(35 \times 12.8 \times$ $1.7 \mathrm{~mm}^{3}$ ) were used for DMA studies. DMA studies were carried out at a frequency of $10 \mathrm{~Hz}$ and at an amplitude $5 \mu \mathrm{m}$ in the temperature range of $30-180{ }^{\circ} \mathrm{C}$.

\section{Results and discussion}

\section{Characterization BPEI- $g$-MWNT}

Amidation reaction between BPEI and carboxylic acid functional groups present on the surface of c-MWNT was characterized using spectroscopic techniques; Fig. 1a shows FTIR spectra, where the characteristic peak for carboxylic group in c-MWNNT is evident at $1700 \mathrm{~cm}^{-1}$, while it has completely 

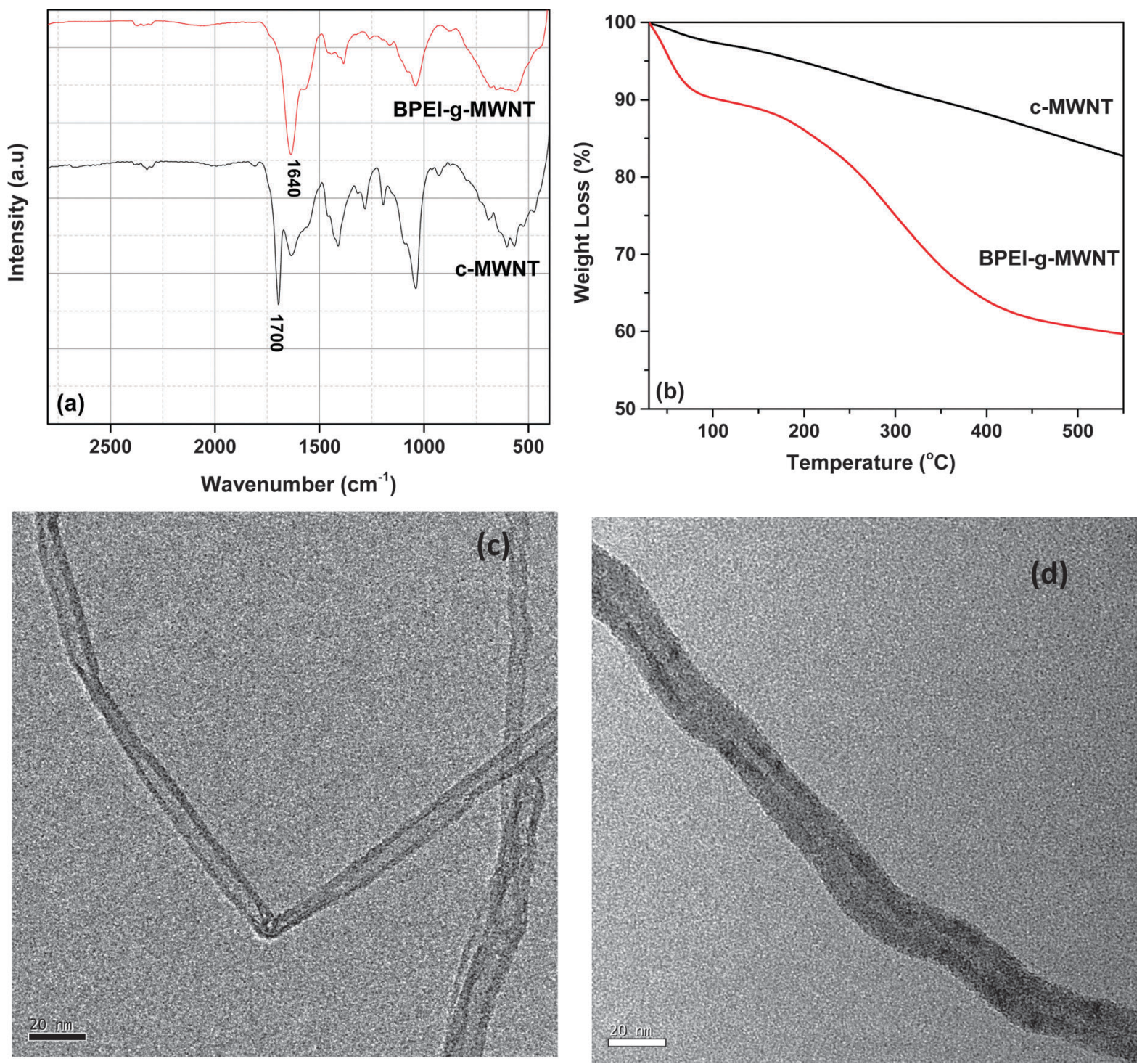

Fig. 1 (a) FTIR spectra of c-MWNT and BPEI-g-MWNT, (b) TGA graph of c-MWNT and BPEI-g-MWNT, (c) and (d) are TEM micrographs of c-MWNT and BPEI-g-MWNT, respectively.

disappeared for BPEI-g-MWNT. In addition, a sharp and intense peak at $1640 \mathrm{~cm}^{-1}$ appeared in the spectra of BPEI-g-MWNT. These two observations clearly demonstrate the successful grafting of BPEI onto MWNTs. TGA was used to evaluate the \% grafting of BPEI. ${ }^{27}$ From the TGA scans (Fig. 1b), the \% residual mass of c-MWNTs at $550{ }^{\circ} \mathrm{C}$ was observed to be $c a .82 \mathrm{wt} \%$, while for BPEI-g-MWNTs, it was observed to be $c a$. 59\%; rapid decomposition of BPEI was observed above $200{ }^{\circ} \mathrm{C}$. Relative weight loss was calculated, which corresponded to the weight percent of BPEI grafted onto MWNTs. It was observed that $c a .28 \%$ of BPEI was grafted on the surface of MWNT. The morphology of the synthesized BPEI-g-MWNT was studied using TEM (Fig. 1c and d). The diameter of the c-MWNT was in the range of 8-10 nm, whereas that of BPEI- $g$-MWNT was found to be between 14-18 nm. Moreover, c-MWNTs were characterized by sharp edges, whereas in the case of BPEI- $g$-MWNTs, a layer along the axis of the MWNT was clearly observed from the TEM micrograph. Both the spectroscopy and the microscopic techniques support the successful grafting of BPEI on the surface of MWNTs. The size of the grafted BPEI chains was calculated using the following expression, where $R_{\mathrm{g}}$ is the radius of gyration and $M_{\mathrm{n}}$ is number average molecular weight of the grafted polymer. ${ }^{28}$ This clearly shows a single layer of BPEI on the surface of MWNTs.

$$
R_{\mathrm{g}}=0.028 M_{\mathrm{n}}^{0.5}=4.43 \mathrm{~nm}
$$

\section{State of dispersion of MWNTs and the fracture surface morphology of epoxy composites}

Fig. 2a-e show the SEM micrographs of the cryo-fractured morphologies of neat epoxy (Fig. 2a) and the various epoxy composites. Fig. $2 \mathrm{~b}$ and c clearly show smaller clusters of c-MWNTs due to the lack of interaction with the matrix as well as due to van der Waals' attractive forces. ${ }^{29}$ However, after 

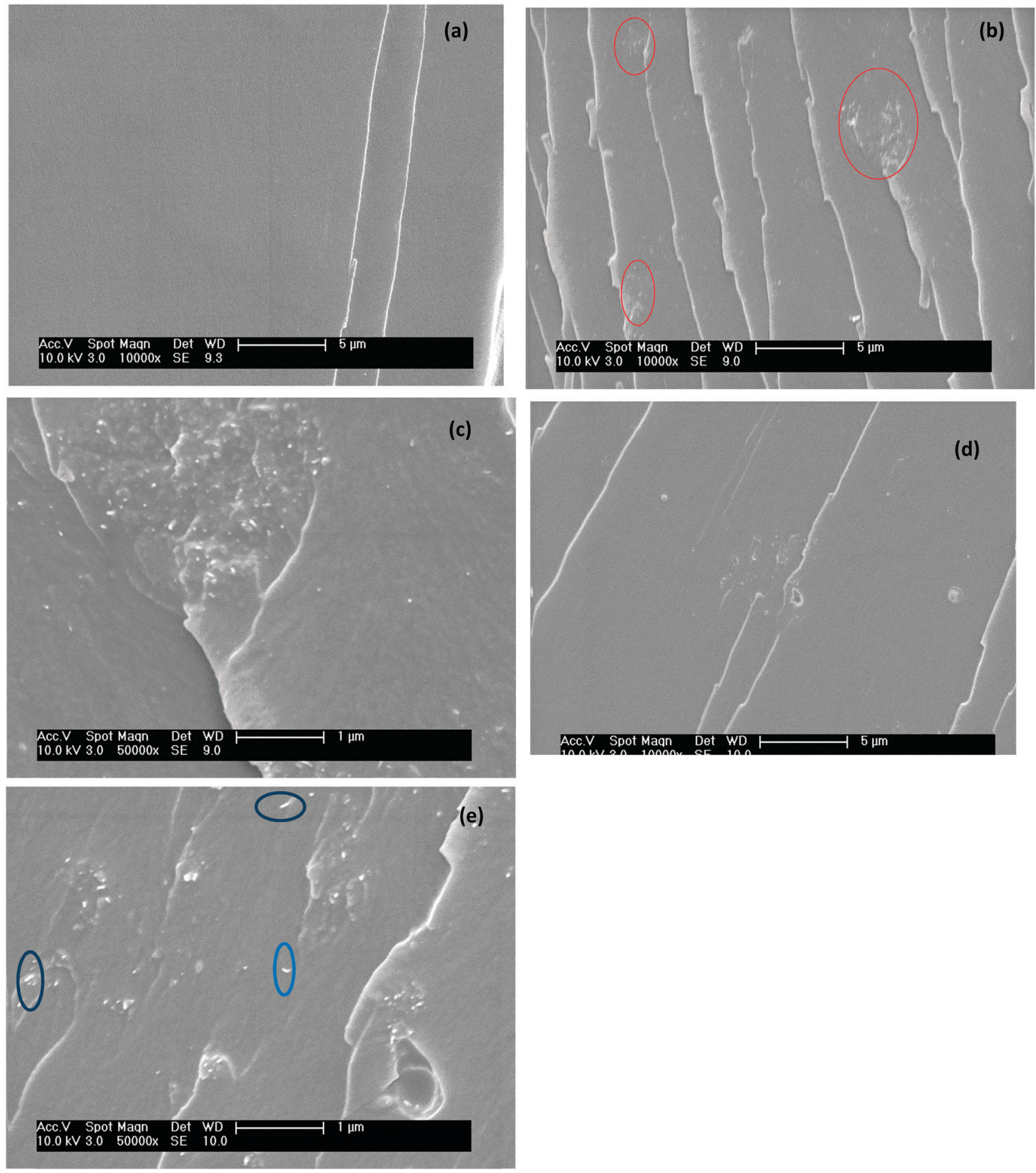

Fig. 2 SEM micrographs of fractured surfaces of epoxy composites: (a) neat epoxy, (b) and (c) 1 wt\% c-MWNT-epoxy composites, (d) and (e) 1 wt\% BPEIg-MWNT-epoxy composites.

functionalization with BPEI, no such features were observed. The BPEI- $g$-MWNT interacts via amine and imine linkages with the epoxy resin leading to better dispersion of MWNTs (Fig. 2d and e). At higher magnification (Fig. 2e), the MWNTs are welldispersed in the matrix. In addition, individual MWNTs were observed in the cryo-fractured morphology of the BPEI- $g$ MWNT-epoxy composites (as highlighted). Peng et al. reported that silane grafted CNTs were dispersed efficiently in epoxy. ${ }^{30}$ In another study, the fluorination of SWNTs resulted in a good dispersion of SWNTs in epoxy matrix. ${ }^{31}$ 


\section{Dynamic mechanical analysis of epoxy composites}

Fig. 3a depicts the storage moduli and tan delta curves for the various composites. It is observed that the addition of different MWNTs improved the mechanical properties of the epoxy resin. For example, 1 wt $\%$ c-MWNTs improved the storage modulus by $20 \%$, while $1 \mathrm{wt} \%$ BPEI- $g$-MWNTs further enhanced the modulus by $49 \%$ with respect to the neat epoxy resin. This can be attributed to the better dispersion and improved interfacial adhesion between BPEI-g-MWNTs and epoxy resin. The BPEI grafted onto MWNTs allows better interaction with the epoxy matrix, and thus allows more cross-linking. This network formation between grafted polymer and matrix assists efficient load transfer. ${ }^{32}$ In addition, it is clearly evident that the storage modulus for c-MWNT-epoxy composite was lower than neat epoxy in the rubbery region, while BPEI- $g$-MWNT-epoxy composite showed a higher storage modulus. Therefore, it can be inferred that BPEI- $g$-MWNT-epoxy composites demonstrate better mechanical properties even in the rubbery region.

From the peak in tan $\delta$ plot, shown in Fig. 3b, the glass transition temperature, $T_{\mathrm{g}}$, which is the temperature at which transition from the glassy to the rubbery state occurs, can be estimated. ${ }^{15,16,33}$
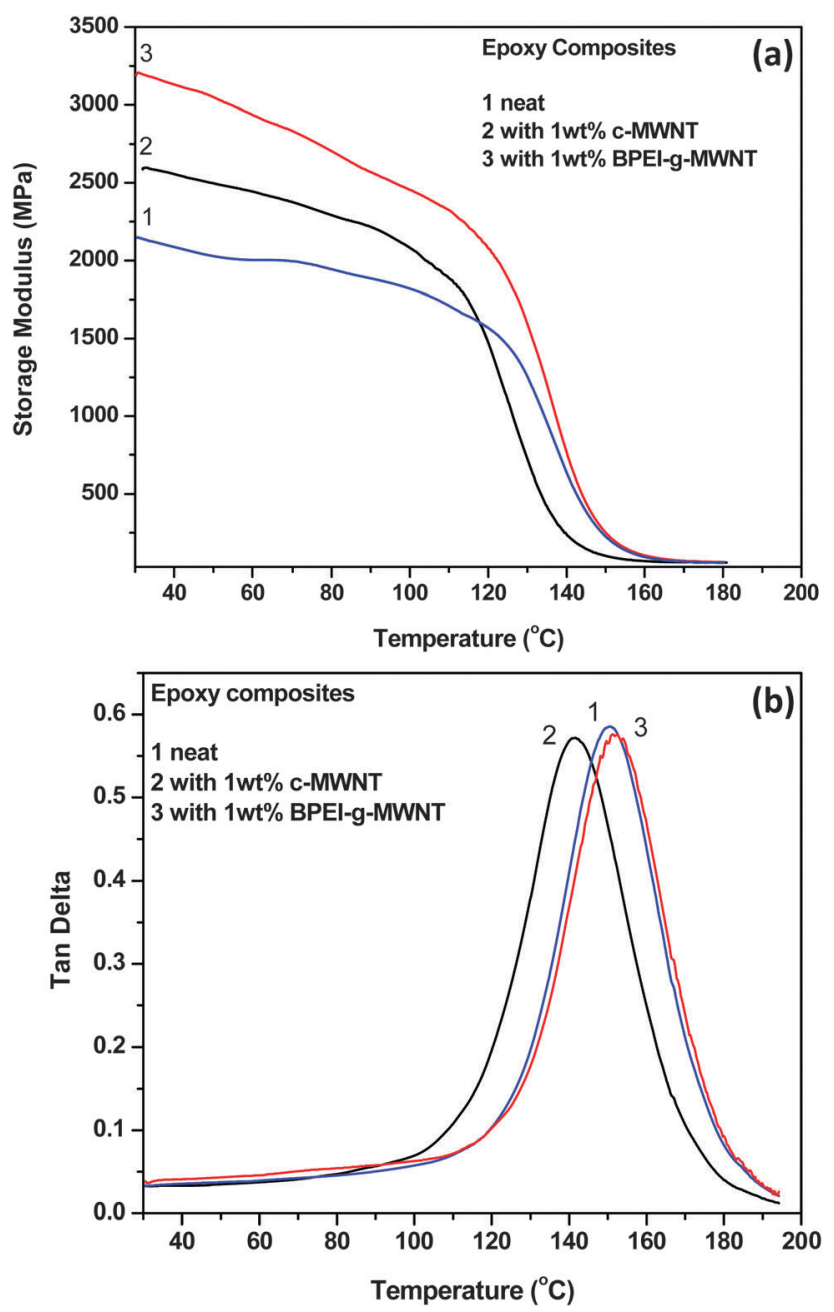

Fig. 3 (a) Storage modulus and (b) Tan delta data for epoxy composites (neat, 1 wt\% c-MWNT, 1 wt\% BPEI-g-MWNT) obtained from dynamic mechanical analyzer.
Interestingly, the $T_{\mathrm{g}}$ of c-MWNTs-epoxy composites was lowered with respect to that of neat epoxy by $7{ }^{\circ} \mathrm{C}$ possibly due to the formation of CNT agglomerates in the matrix, which eventually reduced the cross-linking density. Interestingly, in the presence of BPEI- $g$-MWNTs, the $T_{\mathrm{g}}$ increased by $2{ }^{\circ} \mathrm{C}$, indicating a reduced macromolecular mobility in the presence of exfoliated CNT.

\section{Hardness and elasticity assessment by nanoindentation}

Fig. 4a and b illustrate the load-time cycle (loading, holding and unloading) conditions adopted in this work and the
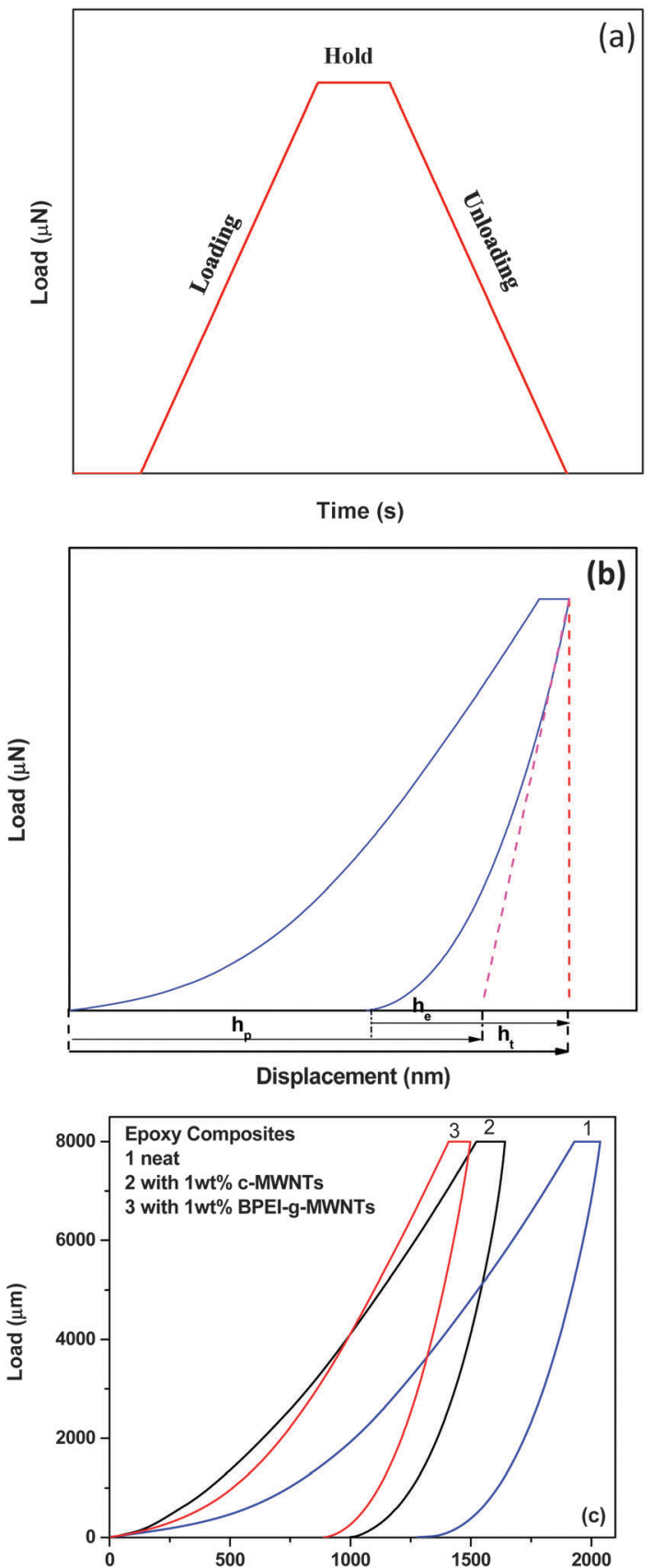

Fig. 4 Representative plots for (a) load as a function of time and (b) load as a function of displacement. (c) Load as a function of displacement for different samples. 
Table 1 Hardness values for the different epoxy composites studied here

\begin{tabular}{llll}
\hline Epoxy composites & $P_{\max }(\mu \mathrm{N})$ & $A\left(\mathrm{~nm}^{2}\right) \times 10^{6}$ & Hardness $(\mathrm{GPa})$ \\
\hline Neat & 8000 & 76.393 & 0.105 \\
With 1 wt\% c-MWNT & 8000 & 47.524 & 0.168 \\
With 1 wt\%BPEI-g-MWNT & 8000 & 40.006 & 0.2
\end{tabular}

Table 2 Plasticity indices of epoxy composites

\begin{tabular}{lll}
\hline Epoxy composites & Plasticity index, $\Psi$ & $\%$ change in $\Psi^{a}$ \\
\hline Neat & 0.58 & - \\
With 1 wt\% c-MWNT & 0.54 & 6.8 \\
With 1 wt\% BPEI- $g$-MWNT & 0.53 & 8.6 \\
&
\end{tabular}

corresponding load-displacement graphs. Fig. $4 \mathrm{c}$ shows the load as a function of displacement for different samples. Table 1 summarizes the hardness value for the various epoxy composites. The hardness, $H$, is calculated from eqn (1), where $P_{\max }$ is the maximum applied load and $A$ is the contact area. The area for the Berkovich indentor is given by eqn (2). ${ }^{20}$ The nanoindentation curve describes the mechanical behaviour of a polymer during loading and unloading cycles, and hence it reveals critical insights into the elastic-plastic behaviour, which is also known as the plasticity index, $\psi$ (eqn (3)). ${ }^{34,35}$

$$
\begin{gathered}
H=\frac{P_{\max }}{A} \\
A=24.5 h_{\mathrm{p}}{ }^{2} \\
\psi=\frac{A_{\mathrm{p}}-A_{\mathrm{e}}}{A_{\mathrm{p}}}
\end{gathered}
$$

It is evident that the addition of different MWNTs enhanced the hardness of the epoxy resin. This observation is in line with the fact that stiffer and stronger nanoparticles enhance the hardness of the polymer matrix. ${ }^{34}$ Therefore, it can be concluded that during the indentation process, the indenter is in contact with stiffer particles and undergoes little plastic deformation. The BPEI-g-MWNTs-epoxy composites showed the highest hardness value among the different composites studied here. As mentioned above, the plasticity index sheds light on the mechanical properties of a material. Moreover, it is a parameter used to analyse the selfhealing ability of a material. ${ }^{34}$ From Table 2 , it can be inferred that there is an improvement of $c a .9 \%$ in the plasticity index of BPEI-g-MWNTs-epoxy composite compared to neat epoxy matrix. Thus, epoxy-BPEI-g-MWNTs shows self-healing behaviour under the given load conditions and can further be explored as a selfhealing material.

\section{Thermal properties}

TGA studies were carried out to study the thermal degradation behaviour in the composites. Fig. 5 depicts the TGA profile for different epoxy composites. It is observed that in all the composites, the degradation started around $300{ }^{\circ} \mathrm{C}$. Therefore, these composites can also be explored for high temperature application. Epoxy composites with BPEI- $g$-MWNTs showed a

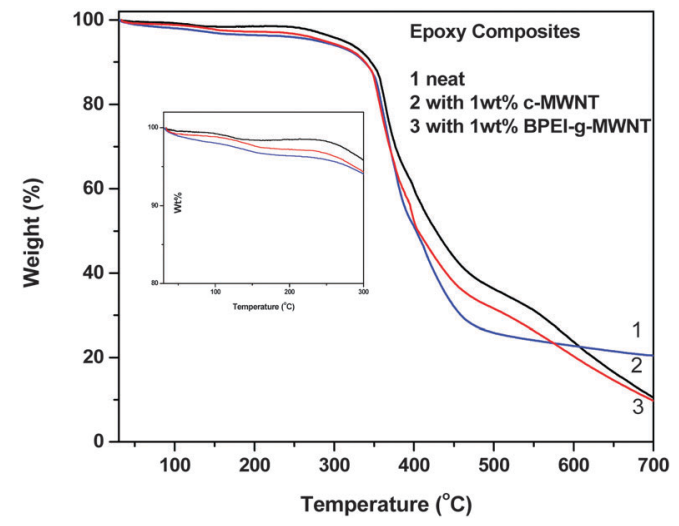

Fig. $5 \%$ weight as a function of temperature from TGA analysis.

small amount of degradation below $300{ }^{\circ} \mathrm{C}$, which can be attributed to the decomposition of BPEI.

\section{Conclusions}

In this study, BPEI- $g$-MWNTs was synthesized using EDC chemistry, which were used to improve the interfacial adhesion, and thus the mechanical behaviour of epoxy matrix was improved using an amine and imine rich molecule. Epoxy composites with $1 \mathrm{wt} \%$ carboxylic acid modified MWNT and BPEI-g-MWNTs were prepared using sonication with mechanical stirring followed by curing and post-curing cycles. The fracture surface morphology of the composites showed better dispersion in the case of BPEI- $g$-MWNTs in epoxy than in the case of c-MWNTs. The storage elastic modulus of BPEI-g-MWNTs-epoxy composites showed $c a$. $49 \%$ improvement with respect to neat epoxy samples and a marginal improvement of $2{ }^{\circ} \mathrm{C}$ in $T_{\mathrm{g}}$. The nanoindentation results displayed increase in the hardness, and hence a lower plastic deformation of epoxy in the presence of $1 \mathrm{wt} \%$ BPEI-g-MWNTs. From the plasticity indices value, it was concluded that BPEI-g-MWNTs-epoxy composites show potential self-healing ability to a certain extent under the given loading conditions. Thermogravimetric analysis showed that the decomposition temperature of all the composites studied here is above $300{ }^{\circ} \mathrm{C}$.

\section{Acknowledgements}

Authors would like to acknowledge the funding agency (DST), Advanced Facility for Microscopy and Microanalysis, IISc (AFMM) for TEM, SEM and Nanoindentation and Mr Prasanna Mural for his assistance in FTIR.

\section{References}

1 S. Iijima, Nature, 1991, 354, 56-58.

2 M. Treacy, T. Ebbesen and J. Gibson, Nature, 1996, 381, 678-680.

3 T. Ebbesen, H. Lezec, H. Hiura, J. Bennett, H. Ghaemi and T. Thio, Nature, 1996, 382, 54-56. 
4 M. Fujii, X. Zhang, H. Xie, H. Ago, K. Takahashi, T. Ikuta, H. Abe and T. Shimizu, Phys. Rev. Lett., 2005, 95, 065502.

5 M. Sharma, M. P. Singh, C. Srivastava, G. Madras and S. Bose, ACS Appl. Mater. Interfaces, 2014, 6, 21151-21160.

6 S. P. Pawar, D. A. Marathe, K. Pattabhi and S. Bose, J. Mater. Chem. A, 2015, 3, 656-669.

7 P. Xavier and S. Bose, RSC Adv., 2014, 4, 55341-55348.

8 R. Rohini and S. Bose, ACS Appl. Mater. Interfaces, 2014, 6, 11302-11310.

9 P. M. Ajayan, O. Stephan, C. Colliex and D. Trauth, Science, 1994, 265, 1212-1214.

10 F.-L. Jin, C.-J. Ma and S.-J. Park, J. Mater. Sci. Eng. A, 2011, 528, 8517-8522.

11 L. Wang, K. Wang, L. Chen, Y. Zhang and C. He, Composites, Part A, 2006, 37, 1890-1896.

12 C. Chen, R. S. Justice, D. W. Schaefer and J. W. Baur, Polymer, 2008, 49, 3805-3815.

13 K. Yang, M. Gu, Y. Guo, X. Pan and G. Mu, Carbon, 2009, 47, 1723-1737.

14 J. Zhu, H. Peng, F. Rodriguez-Macias, J. L. Margrave, V. N. Khabashesku, A. M. Imam, K. Lozano and E. V. Barrera, Adv. Funct. Mater., 2004, 14, 643-648.

15 Y. Geng, M. Y. Liu, J. Li, X. M. Shi and J. K. Kim, Composites, Part A, 2008, 39, 1876-1883.

16 M. Yoonessi, M. Lebrón-Colón, D. Scheiman and M. A. Meador, ACS Appl. Mater. Interfaces, 2014, 6, 16621-16630.

17 W. Zou, Z.-j. Du, Y.-x. Liu, X. Yang, H.-q. Li and C. Zhang, Compos. Sci. Technol., 2008, 68, 3259-3264.

18 H. Lee, S. Mall, P. He, D. Shi, S. Narasimhadevara, Y.-H. Yun, V. Shanov and M. J. Schulz, Composites, Part B, 2007, 38, 58-65.

19 J. A. Ramos, M. Blanco, I. Zalakain and I. Mondragon, J. Colloid Interface Sci., 2009, 336, 431-437.
20 A. M. Díez-Pascual, M. A. Gómez-Fatou, F. Ania and A. Flores, Prog. Mater. Sci., 2015, 67, 1-94.

21 S. Chen and J. Feng, Compos. Sci. Technol., 2014, 101, 145-151.

22 S. Movva, X. Ouyang, J. Castro and L. J. Lee, J. Appl. Polym. Sci., 2012, 125, 2223-2230.

23 D. Schaubroeck, J. De Baets, T. Desmet, P. Dubruel, E. Schacht, L. Van Vaeck and A. Van Calster, Appl. Surf. Sci., 2010, 256, 6269-6278.

24 J. Huang, M. Gao, T. Pan, Y. Zhang and Y. Lin, Compos. Sci. Technol., 2014, 95, 16-20.

25 H. Kim, R. Namgung, K. Singha, I.-K. Oh and W. J. Kim, Bioconjugate Chem., 2011, 22, 2558-2567.

26 B. Chen, M. Liu, L. Zhang, J. Huang, J. Yao and Z. Zhang, J. Mater. Chem., 2011, 21, 7736-7741.

27 P. K. S. Mural, M. S. Rana, G. Madras and S. Bose, RSC Adv., 2014, 4, 16250-16259.

28 G. P. Kar, P. Xavier and S. Bose, Phys. Chem. Chem. Phys., 2014, 16, 17811-17821.

29 L. Yue, G. Pircheraghi, S. A. Monemian and I. ManasZloczower, Carbon, 2014, 78, 268-278.

30 P. C. Ma, J.-K. Kim and B. Z. Tang, Compos. Sci. Technol., 2007, 67, 2965-2972.

31 J. Zhu, J. Kim, H. Peng, J. L. Margrave, V. N. Khabashesku and E. V. Barrera, Nano Lett., 2003, 3, 1107-1113.

32 G. L. Hwang, Y. T. Shieh and K. C. Hwang, Adv. Funct. Mater., 2004, 14, 487-491.

33 L. Sun, G. Warren, J. O'reilly, W. Everett, S. Lee, D. Davis, D. Lagoudas and H.-J. Sue, Carbon, 2008, 46, 320-328.

34 M. G. Ahangari and A. Fereidoon, Mater. Chem. Phys., 2014, 151, 112-118.

35 M. Munz, J. Phys. D: Appl. Phys., 2006, 39, 4044. 